\title{
Rapid on-site Detection of Illicit Drugs in Smuggled Samples with a Portable Electrochemical Device
}

\author{
Marc Parrilla 1,2, Amorn Slosse 3, Robin Van Echelpoel 1,2, Noelia Felipe Montiel 1,2, Amelia R. Langley 1,2, Filip Van \\ Durme $^{3}$ and Karolien De Wael ${ }^{1,2, *}$
}

1 A-Sense Lab, Department of Bioscience Engineering, University of Antwerp, Groenenborgerlaan 171, 2020, Antwerp, Belgium; marc.parrillapons@uantwerpen.be (M.P.); robin.vanechelpoel@uantwerpen.be (R.V.E.); noelia.felipemontiel@uantwerpen.be (N.F.M.); amelia.langley@uantwerpen.be (A.R.L.)

2 NANOlab Center of Excellence, University of Antwerp, Groenenborgerlaan 171, 2020, Antwerp, Belgium.

3 Drugs and Toxicology Department, National Institute for Criminalistics and Criminology (NICC), Vilvoordsesteenweg 100, 1120, Brussels, Belgium; amorn.slosse@just.fgov.be (A.S.); filip.vandurme@just.fgov.be (F.V.D.)

* Correspondence: karolien.dewael@uantwerpen.be; Tel.: (+32 32653335)

\begin{abstract}
Illicit drug consumption is posing critical concerns in our society causing health issues, crime-related activities, and the disruption of border trade. The smuggling of illicit drugs urges the development of new tools for rapid on-site identification in cargos. Current methods used by law enforcement officers rely on presumptive color tests and portable spectroscopic techniques. However, these methods sometimes exhibit inaccurate results due to commonly used cutting agents, the colorful nature of the sample or because the drugs are smuggled (hidden or mixed) in common goods. Interestingly, electrochemical sensors can deal with these specific problems. Herein, an electrochemical device is presented that uses affordable screen-printed electrodes for the electrochemical profiling of illicit drugs by square-wave voltammetry (SWV). The identification of the illicit compound is based on the oxidation potential of the analyte. Hence, a library of electrochemical profiles is built upon the analysis of illicit drugs and common cutting agents. This library allows the design of a tailor-made script that enables the identification of each drug through a user-friendly interface (laptop or mobile phone). Importantly, the electrochemical test is compared by analyzing 48 confiscated samples with other portable devices based on Raman and FTIR spectroscopy as well as a laboratory standard method (i.e. gas chromatography - mass spectrometry). Overall, the electrochemical results obtained through the analysis of different samples from confiscated cargos at an enduser site, present a promising alternative to current methods, offering low-cost and rapid testing in the field.
\end{abstract}

Keywords: Electrochemical sensors; square-wave voltammetry; screen-printed electrodes; electrochemical profiling, illicit drugs; forensic analysis; Raman spectrometer, FTIR spectrometer; on-site detection

\section{Introduction}

The consumption of drugs of abuse is causing critical issues in our society due to health issues, crime-related activities, and border trade disruption [1,2]. Still, around 83 million, or $28.9 \%$, of adults (aged 15-64) are estimated to have used illicit drugs at least once in their lifetime in the European Union [1]. In the last year, 3.5 million adults used cocaine, 2.6 million adults used 3,4-methylenedioxymethamphetamine (MDMA), and 2.0 million adults used amphetamines (i.e. amphetamine and methamphetamine), highlighting the high proportion of the EU population at risk [1]. Moreover, 1.0 million people are identified as high-risk opioid users, with opioids being the cause of $76 \%$ of all fatal illicit drug overdoses [1]. Therefore, there is a societal need for decreasing the consumption of illicit drugs. One option is to avoid illicit drugs reaching the market by: (i) controlling the 
entrance of illicit drugs, (ii) blocking manufacturing settings, and (iii) raising awareness about the risks among the general public.

Illicit drugs enter the illegal market through external borders (e.g. natural drugs) or by internal production (e.g. synthetic drugs), despite the difficulties in trading during the COVID-19 pandemic [3]. On one side, the smuggling of illicit drugs such as cocaine and heroin in Europe urges the development of new tools for rapid on-site identification in cargos. On the other side, the production of synthetic drugs increases internal trafficking, thus demanding simple and straightforward devices to detect illicit drugs in the field. Overall, a fast method for the on-site detection of illicit drugs seems a suitable option to control illegal trading.

Current methods used by law enforcement officers rely on presumptive color tests [4,5] and portable spectroscopic techniques [6-8]. Recently, miniaturized devices employing near-infrared $[9,10]$ have been developed showing promise for the rapid on-site detection of illicit drugs with the potential to overcome critical limitations. However, current methods used by border officers sometimes exhibit inaccurate results due to commonly used cutting agents or because the drug samples are colored [11]. Particularly for presumptive color tests, interpretation of the colors is subjective, and samples that are colored might also influence the test's results $[12,13]$. Concerning spectroscopic techniques, such equipment remains expensive and the analysis of dark or colored samples can be challenging due to fluorescence interference, as in the case of Raman spectrometers [14]. Besides, drug traffickers are generating innovative ways to overcome traditional detection methods, such as mixing with conventional goods (e.g. charcoal, food), or adding colorants or other substances to avoid on-site determination by current methods. Therefore, new devices that can overcome the current problems are necessary to cope with the determination of smuggled illicit drugs in common goods.

The devices for on-site analysis must be portable, low-cost, and user-friendly to be implemented and used by law enforcement officers [15]. Electrochemical sensors can provide the aforementioned features and, importantly, they can deal with current challenges, providing more reliable results in comparison to commercially available devices [16-18]. In this direction, portable and wearable electrochemical sensors have been designed for the detection of illicit drugs using different configurations such as a glove-based sensor [19] as well as for their determination in biofluids [20]. The electrochemical approach is based on the characteristic electrochemical profile of each compound that reveals the electroactive functionalities of the target compound [21]. Following this strategy, cocaine $[12,22]$, ketamine [23], heroin [24] and synthetic cathinones [25,26] have been detected by using low-cost screen-printed electrodes (SPEs). Amphetamine is a special case as it is not electroactive in the potential window of commercial carbon SPEs. Therefore, in situ derivatization is required by employing 1,2-Naphthoquinone-4-sulphonic acid sodium salt, allowing its electrochemical detection [27]. Overall, the most used illicit drugs can be detected by electrochemical methods under certain conditions.

Herein, an electrochemical device is presented that uses low-cost SPEs for the electrochemical detection of illicit drugs by square-wave voltammetry (SWV) profiling. The combination of preliminary visual identification followed by an electrochemical test allows the detection of commonly encountered illicit drugs in Europe (i.e. cocaine, MDMA, heroin, amphetamine and, methamphetamine). The visual identification permits the selection of a suitable $\mathrm{pH}$ strategy for the electrochemical test which will unravel the particular profile of each illicit drug. The electrochemical interrogation of the illicit drugs provides characteristic oxidation peak potentials for electroactive moieties present in each drug, except for amphetamine which needs in situ derivatization to unravel its characteristic oxidation peak potential. A library of electrochemical profiles is built upon pure illicit drugs with their respective common cutting agents. This library allows the design of a tailor-made script that shows the identification of each drug according to each oxidation potential through a user-friendly interface. Finally, the electrochemical device is validated with confiscated samples and compared with standard methods from forensic 
laboratories (e.g. gas chromatography-mass spectrometry). Interestingly, a benchmarking study with common spectroscopic techniques is also provided. Overall, the fast analysis of samples with a portable electrochemical device provides a straightforward on-site detection method aiming to facilitate the tasks of law enforcement officers in the field, thus providing more secure border management and a safer society.

\section{Methods}

\subsection{Materials}

Standards of D, L-amphetamine $\cdot \mathrm{HCl}$, methamphetamine $\cdot \mathrm{HCl}, 3$,4-methylenedioxymethamphetamine $\cdot \mathrm{HCl}(\mathrm{MDMA})$, cocaine $\cdot \mathrm{HCl}$ and heroin $\cdot \mathrm{HCl}$, were purchased from Chiron AS, Norway. Standards of paracetamol, phenacetin, levamisole, lidocaine, caffeine, benzocaine, procaine, lactose and creatine were provided by the National Institute for Criminalistics and Criminology (NICC, Belgium). Confiscated samples of amphetamine, MDMA, cocaine and heroin were also provided by the NICC. Analytical grade salts of potassium chloride, potassium phosphate, sodium borate, sodium bicarbonate, sodium acetate and potassium hydroxide were purchased from Merck Sigma-Aldrich (Overijse, Belgium). 1,2- naphthoquinone-4-sulphonic acid sodium salt (NQS) (>98\%) was purchased from Tokyo Chemical Industry Co., LTD., Japan. All solutions were prepared in 18.2 $\mathrm{M} \Omega \mathrm{cm}^{-1}$ doubly deionized water (Milli-Q water systems, Merck Millipore, Germany). The $\mathrm{pH}$ was measured using a pH-meter (914 pH/Conductometer, 2.914.0020, Metrohm, Switzerland).

\subsection{Methods}

Square-wave voltammograms (SWVs) were recorded using a MultiPalmSens4 or EmStat Pico potentiostats (PalmSens, The Netherlands) with PSTrace/MultiTrace. Disposable ItalSens SPEs (PalmSens, the Netherlands), containing a graphite working electrode (diameter, $\varnothing=3 \mathrm{~mm}$ ), a carbon counter electrode, and a (pseudo) silver reference electrode were used for all measurements. The SWV parameters used were: potential range of 0.0-1.4 V, frequency $10 \mathrm{~Hz}, 25 \mathrm{mV}$ amplitude and $5 \mathrm{mV}$ step potential. All voltammograms shown were background corrected using the "moving average iterative background correction" (peak width =1) tool in the PSTrace software.

Electrochemical tests were performed in $20 \mathrm{mM}$ buffer solutions with $100 \mathrm{mM} \mathrm{KCl}$ by applying $60 \mu \mathrm{l}$ of the solution onto the SPE. Phosphate buffer (PBS) (buffer A), acetate buffer (buffer B) and hydrogen carbonate buffer (buffer C) were used for the detection of cocaine and heroin, MDMA and amphetamine, respectively. Preanodized SPEs for heroin detection were performed by applying $1.5 \mathrm{~V}$ for $60 \mathrm{~s}$ in PBS solution at $\mathrm{pH} 7$ by drop casting $60 \mu \mathrm{L}$ on the SPE [24].

The composition of the confiscated samples was previously analyzed in the forensic laboratory at NICC with standard methods to subsequently validate the electrochemical approach. The qualitative analysis of the street samples was performed using gas chromatography-mass spectrometry (GC-MS), Agilent1 6890 N gas chromatographic system (Agilent Technologies, Santa Clara, CA, USA) coupled to an Agilent1 5973 N mass selective detector [28]. The applied chromatographic methods are ISO17025 accredited and are continuously evaluated through participation in international quality control programs (United Nations Office on Drugs and Crime - UNODC, and European Network of Forensic Science Institutes - ENFSI).

A Bruker Bravo Handheld Raman spectrometer (Bruker Optik GmbH, Ettlingen, Germany) was used for all Raman measurements. The instrument uses a dual laser excitation feature with two laser diodes (wavelengths: $785 \mathrm{~nm}$ and $852 \mathrm{~nm}$ ). Spectra were recorded from $170 \mathrm{~cm}^{-1}$ to $3200 \mathrm{~cm}^{-1}$. OPUS 8.2.28 (Bruker Optik $\mathrm{GmbH}$, Ettlingen, Germany) software was used for data acquisition and analysis. All seized samples were processed into powdered form and stored in transparent plastic bags. All measurements were performed by placing the plastic bag containing the sample on the measuring tip. Identification was 
performed using the TICTAC Drug Library (TICTAC Communications Ltd., London, United Kingdom).

Attenuated total reflectance (ATR) Fourier transform infrared (FTIR) (Bruker Alpha II spectrometer, Bruker Optik GmbH, Ettlingen, Germany) was used for the analysis of the confiscated samples, employing a diamond crystal. For each measurement, a small amount of sample was placed directly on the crystal. The spectra were recorded from 4000 $\mathrm{cm}^{-1}$ to $400 \mathrm{~cm}^{-1}$ with a spectral resolution of $4 \mathrm{~cm}^{-1}$ and consisting of 128 co-added scans (analysis time: ca. 170 s). A background scan (128 scans) was run against air before the measurements. Data acquisition and analysis were also performed using OPUS 8.2.28 software. The TICTAC Drug Library (for ATR spectra) was used for identification.

For the electrochemical analysis of the seized samples (Figure 1), first, visual identification of the sample was performed regarding the color and type of sample (e.g. powder, crystal, pill) (Figure 1A) and, second, according to the physical appearance of the sample, a buffer was selected for the electrochemical test (Figure 1B). This test uses a SPE on a portable potentiostat connected to a laptop via Bluetooth ${ }^{\circledR}$ or cable. The interface launches the electrochemical analysis and displays the output of the identification (Figure 1C). The obtained SWV was background corrected using the "moving average iterative background correction" (peak width $=1$ ) tool and subsequently digitally filtered with a top-hat filter $(\mathrm{wt}=7)$. This digital filtering and all further pre-processing steps were executed utilizing an in-house developed MATLAB script (Matlab R2018b, MathWorks, U.S.A.). After the pre-processing of the voltammograms, the relevant peaks were selected based on a minimum peak prominence and minimum peak height threshold. Subsequently, compounds were assigned to the selected peaks using an internal library. A detailed description of this data processing approach can be found elsewhere [29].

Regarding sample preparation, a disposable microspatula was used to sample ca. $5 \mathrm{mg}$ of the suspicious powder or solution and deposited onto a $15 \mathrm{~mL}$ tube containing the suitable buffer, and thoroughly mixed for $30 \mathrm{~s}$, reaching a concentration of ca. $0.3 \mathrm{mg} \mathrm{mL}^{-1}$ corresponding to $1-22 \mathrm{mM}$ of the pure substance. Considering that the encountered powders were not $100 \%$ pure, lower concentrations of the illicit drug in the sample were expected. Finally, a $60 \mu \mathrm{L}$ drop of the solution was placed on the SPE surface utilizing a disposable pipette for the electrochemical analysis by SWV. The process took approximately $1 \mathrm{~min}$ to sample and $1 \mathrm{~min}$ for the electrochemical output.



Figure 1. Flowchart towards the electrochemical test consisting of: A) visual identification of the suspicious sample, B) selection of the conditions for the electrochemical analysis, and C) output of the identification through the tailor-made script.

\section{Results and Discussion}

\subsection{Electrochemical profiling of illicit drugs}

The electrochemical profiling is based on the interrogation by SWV of the target molecules, which exhibit an oxidation process at characteristic potentials [30]. Fortunately, 
illicit drugs contain moieties that are electroactive within the potential window of carbon SPEs. These moieties are usually secondary or tertiary amines that allow their oxidation, thus showing a peak signal during the anodic electrochemical scan by SWV. For example, the cocaine oxidation peak potential might differ from heroin oxidation peak potential, making it possible to identify the target according to their characteristic peak potentials $\left(\mathrm{E}_{\mathrm{p}}\right)$. However, some illicit drugs share some electroactive moieties whose oxidation peaks overlap, making it difficult to identify characteristic peak potentials.

In previous works, our research group has optimized the detection of illicit drugs by exploring certain conditions during the SWVs. For example, anodic pretreatment of SPEs allows the elucidation of phenolic group oxidation associated with 6-monoacetylmorphine (6-MAM) (a byproduct of heroin degradation at $\mathrm{pH} 12$ ) which would otherwise overlap with the oxidation of paracetamol (a common adulterant [24]). Cathodic pretreatment on the other hand can suppress the effects of some adulterants [25], also achieved by the use of different buffer $\mathrm{pH}$ (as some moieties are not oxidizable at certain $\mathrm{pH}$ levels at SPEs due to their protonation) [23]. Some illicit drugs, such as amphetamine, need a derivatization step to allow its electrochemical profiling when employing low-cost carbon SPEs. In this case, a simple mixing step with NQS initiates a chemical reaction, producing an electroactive product at carbon SPEs [27].

In previous works, a $\mathrm{pH}$ and buffer/electrolyte screening was performed for each target drug (i.e. cocaine [12], heroin [24], amphetamine [27], MDMA [13]) to obtain optimal conditions for the electrooxidation of the drug at carbon SPEs. Acetate buffer at $\mathrm{pH} 5$ and phosphate buffer $\mathrm{pH} 12$ solutions were subsequently identified as optimal for each target drug other than for the derivatization of amphetamine, where hydrogen carbonate buffer at $\mathrm{pH} 10$ was identified as optimal. Furthermore, the use of anodic pretreated SPEs (p-SPE) for the detection of heroin and the carbon SPEs for the rest of the illicit drugs were selected to be used as disposable sensors. Therefore, the aforementioned buffers were employed for the construction of the library on SPE and p-SPE which is integrated into the script database for the identification of illicit drugs. Overall, the use of the flowchart (Figure 1) consisting of a preliminary visual identification ensures the selection of the suitable electrochemical test (i.e. buffer solution and SPE), of which the results are subsequently analysed by the database and displayed on the interface (i.e. positive or negative for the illicit drug along with the electrochemical profile, Figure S1).

A) SPE pH 12

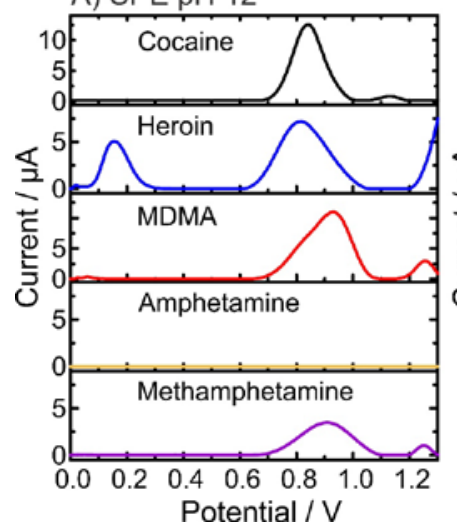

B) Preanodized SPE pH 12

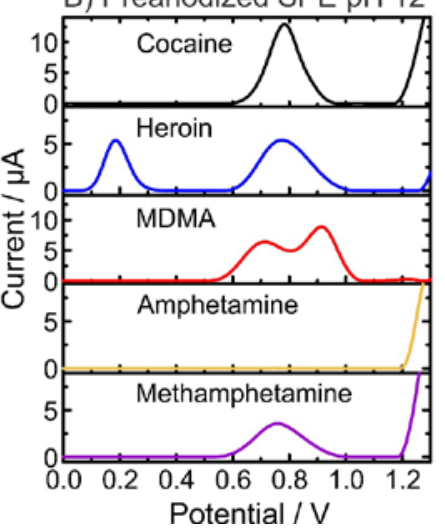

C) SPE pH 5

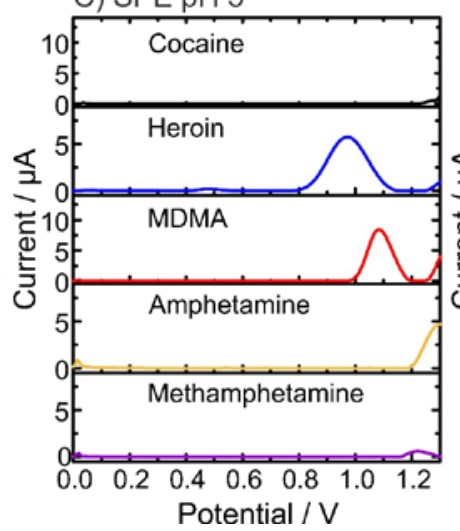

D) SPE pH $10+$ NQS

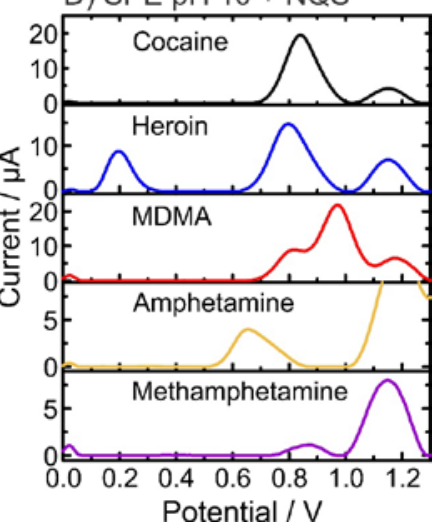

Figure 2. Electrochemical profiles of illicit drugs $(0.5 \mathrm{mM})$ obtained by square-wave voltammetry (SWV) using SPEs and buffers at different $\mathrm{pH}$ values: A) pH 12; B) pH 12 using p-SPE; $\mathrm{pH}$ 5; and pH 10 including the derivatizing agent NQS.

\subsection{Generating the library of electrochemical profiles}

Figure 2 shows the SWVs for $0.5 \mathrm{mM}$ pure illicit drugs (cocaine, heroin, MDMA, amphetamine, and methamphetamine) at $\mathrm{pH} 5, \mathrm{pH} 10, \mathrm{pH} 12$ on carbon SPE, and $\mathrm{pH} 12$ using p-SPEs to record the characteristic electrochemical profiles of the molecules at certain 
conditions. Figure 2A and Figure 2B show the differences between SPEs and p-SPEs in PBS $\mathrm{pH}$ 12. Although similar profiles were obtained, clear peak separation occurred for the MDMA signal, moving from a shoulder at SPE/pH 12 (ca. $0.8 \mathrm{~V}$ ), to a well-defined peak at $\mathrm{p}-\mathrm{SPE} / \mathrm{pH} 12\left(E_{\mathrm{p}}=0.71 \mathrm{~V}\right)$. The MDMA $E_{\mathrm{p}}$ is attributed to the oxidation of the secondary amine. The second oxidation peak (i.e. $E_{\mathrm{p}}=0.91 \mathrm{~V}$ ), which does not change with the use of p-SPE, is attributed to the methylenedioxy group of MDMA [31]. Concerning the other drugs, electrochemical oxidation contributions are attributed to amine-containing groups (i.e. tertiary amines for heroin and cocaine, and secondary amine for methamphetamine and MDMA) which all shifted by 60 to $100 \mathrm{mV}$ towards negative potentials, similar to the first peak of MDMA. The $\mathrm{pH}$ dependence for the oxidation of illicit drugs is clearly shown during analysis at $\mathrm{pH} 5$, where only heroin $\left(E_{\mathrm{p}}=0.97 \mathrm{~V}\right)$ and MDMA $\left(E_{\mathrm{p}}=1.08 \mathrm{~V}\right)$ exhibited electroactivity (Figure $\left.2 \mathrm{C}\right)$. Such $\mathrm{pH}$ dependency can assist in the proper identification of the unknown sample by a simple dual $\mathrm{pH}$ test. Finally, the $\mathrm{pH}$ assessment clearly demonstrates that amphetamine is not electroactive, and only after the derivatization step (Figure 2D), an oxidation peak appears $\left(E_{\mathrm{p}}=0.66 \mathrm{~V}\right)$. Importantly, the derivatization step barely changes the $E_{\mathrm{p}}$ of the other illicit drugs, thus allowing the differentiation of the amphetamine $E_{\mathrm{p}}$ in comparison to other illicit drugs. An oxidation peak was observed at $1.15 \mathrm{~V}$ due to NQS oxidation at the carbon SPE. Since this occurs outside the potential window for illicit drugs (from 0.6 to $1.03 \mathrm{~V}$ ) in $\mathrm{pH} 10$ it will not affect their identification. Table 1 provides all oxidation peaks corresponding to each illicit drug in certain conditions.
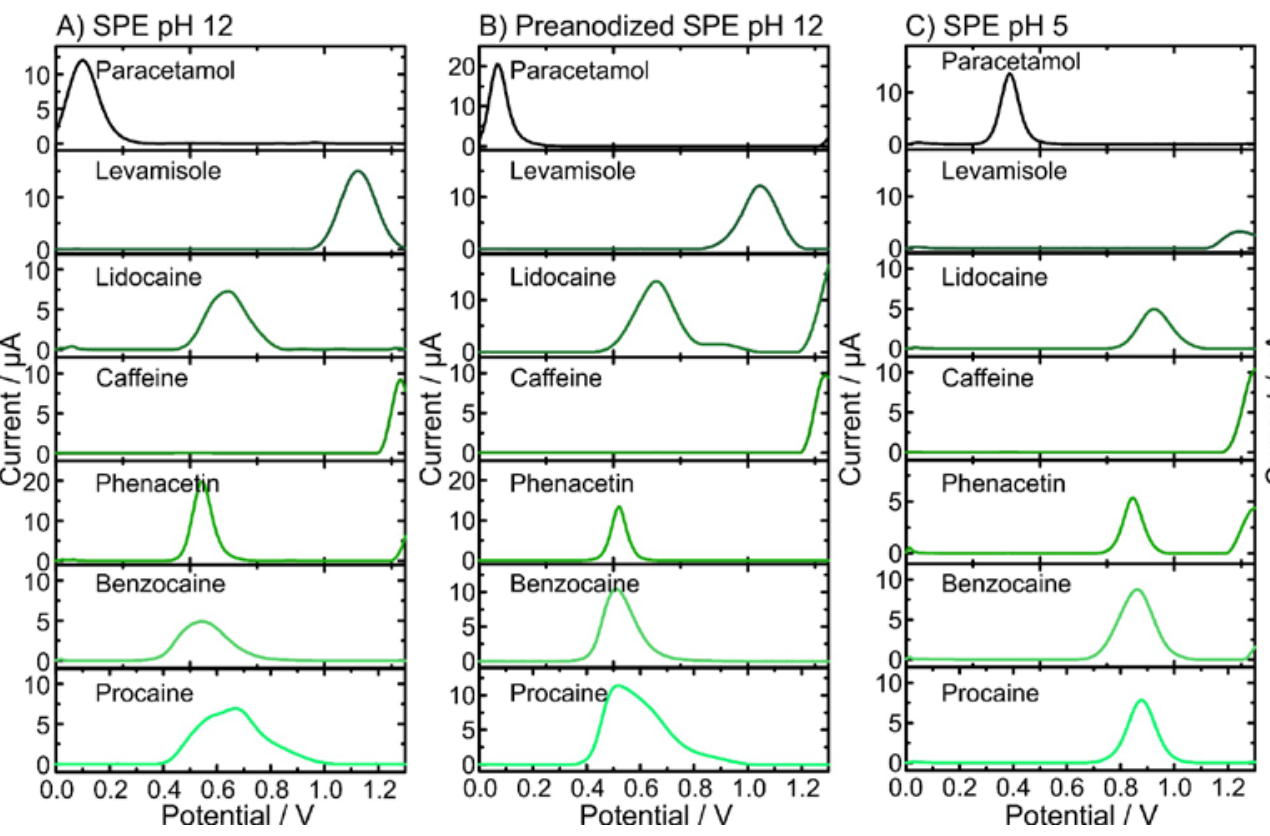

\section{D) SPE pH $10+\mathrm{NQS}$}



Figure 3. Electrochemical profiles of common cutting agents $(0.5 \mathrm{mM})$ obtained by square-wave voltammetry (SWV) using SPE at different $\mathrm{pH}$ : A) $\mathrm{pH}$ 12; B) $\mathrm{pH} 12$ using preanodized SPE; $\mathrm{pH}$ 5; and $\mathrm{pH} 10$ including the derivatizing agent NQS.

A similar electrochemical approach was performed employing the most encountered cutting agents in the reported illicit drugs (i.e. paracetamol, levamisole, lidocaine, caffeine, phenacetin, benzocaine, procaine, and lactose [24,27,32,33]) (Figure 3A-D). In alkaline conditions (i.e. pH 12) (Figure 3A), paracetamol exhibited the most negative oxidation peak corresponding to its phenolic group oxidation (ca. $0.1 \mathrm{~V}$ ). In contrast, caffeine oxidized at high potential values (i.e. $>1.3 \mathrm{~V}$ ), outside the electrochemical profiling of interest. However, there are a set of compounds that displayed oxidation signals near the potential window of interest for illicit drug detection (from $0.76 \mathrm{~V}$ to $0.93 \mathrm{~V}$ in $\mathrm{pH} 12$ ) that must be considered. Phenacetin, benzocaine, and procaine should not interfere with the detection of cocaine as their respective $E_{\mathrm{p}}$ values are located far from the $E_{\mathrm{p}}$ of cocaine. 
Lidocaine and levamisole $E_{\mathrm{p}}$ are closer to the $E_{\mathrm{p}}$ of cocaine but do not overlap [12]. Similar to cocaine, heroin should be able to be detected via a two-peak recognition strategy. However, as paracetamol (one of the most used adulterants in heroin) might overlap with the first peak of heroin $(0.09 \mathrm{~V}$ and $0.16 \mathrm{~V}$, respectively), an additional strategy was required. The anodic pretreatment of the SPE led the paracetamol and heroin signals to shift in $E_{\mathrm{p}}$ to $0.07 \mathrm{~V}$ and $0.19 \mathrm{~V}$, respectively (Figure 3B). This permits the proper identification of the 6-MAM peak, thus avoiding peak overlap [24]. Furthermore, $\mathrm{pH}$ dependence was observed during the analysis at $\mathrm{pH} 5$, where the oxidation peaks for heroin and MDMA shifted towards higher potentials. Importantly, the oxidation peaks of the cutting agents did not overlap with the potential window of illicit drug detection at $\mathrm{pH} 5$ (from $0.97 \mathrm{~V}$ to $1.1 \mathrm{~V}$ ) (Figure 3C). As pH 10 with NQS is targeted for the detection of amphetamine, only common cutting agents encountered in amphetamine real samples were explored (e.g. caffeine, creatine, paracetamol, and lactose $[11,27]$ ) (Figure 3D). Lactose and creatine, mainly found in amphetamine seizures, do not exhibit any redox processes at SPEs within the illicit drug detection window. For paracetamol, its oxidation peak was not located in the detection potential window of amphetamine (from 0.6 to $0.72 \mathrm{~V}$ ). After profiling the cutting agents, most of the $E_{\mathrm{p}}$ did not interfere with $E_{\mathrm{p}}$ values for illicit drugs, thus allowing for a suitable identification in real samples (Table 1).

Table 1. Peak potentials of each target compound at the specific condition (i.e. buffer and SPE).

\begin{tabular}{ccccc}
\hline Target compound / & SPE pH 12 & SPE p-SPE & SPE pH 5 & SPE pH 10+NQS \\
$\boldsymbol{E}_{\mathbf{p}}(\mathrm{V})$ & 0.85 & 0.78 & - & 0.84 \\
Cocaine & $0.16,0.81$ & $0.19,0.77$ & 0.97 & $0.20,0.80$ \\
Heroin & 0.93 & $0.71,0.91$ & 1.08 & $0.82,0.97$ \\
MDMA & - & - & - & 0.66 \\
Amphetamine & 0.91 & 0.76 & - & 0.87 \\
Methamphetamine & 0.09 & 0.07 & 0.39 & - \\
Paracetamol & 1.12 & 1.04 & 1.25 & - \\
Levamisole & 0.65 & 0.66 & 0.92 & - \\
Lidocaine & - & - & - & - \\
Caffeine & 0.54 & 0.52 & 0.85 & - \\
Phenacetin & 0.55 & 0.52 & 0.86 & - \\
Benzocaine & 0.67 & 0.52 & 0.88 & \\
Procaine & & &
\end{tabular}

After building the library of electrochemical profiles from the square-wave analysis of the target illicit drugs in several conditions, the values of $E_{p}$ for each compound at each condition (Table 1) were selected and integrated into a custom-made script. In order to avoid false negatives due to shifts in the $E_{\mathrm{p}}$ within the analysis conditions, a potential window for each target compound was determined. This potential window attempts to enclose the oxidation potential of the illicit drug despite different concentrations, small changes in $\mathrm{pH}$ and/or shifts due to adulterants. Therefore, a potential window within $\pm 60 \mathrm{mV}$ of the $E_{\mathrm{p}}$ of each illicit drug was employed. The script enhances peak separation and facilitates the identification of illicit drugs in the sample. In brief, the script has three functionalities: (i) first the removal of the background signal; (ii) second it applies a tophat filter that provides an enhanced separation of overlapped peaks; and lastly, (iii) it permits the successful identification of substances based on the $E_{\mathrm{p}}$ of each drug by displaying the output of the electrochemical test matching positive or negative detection for the illicit drug. Importantly, the filter is essential when several oxidation peaks are displayed in the same profile caused by a mixture of illicit drugs and adulterants [29]. Because of the proximity of the $E_{p}$ of adulterants and illicit drugs, a shoulder or peak overlap might occur. Hence, the filter facilitates the separation of these peaks allowing for a proper identification based on the $E_{\mathrm{p}}$ window. Therefore, as previously mentioned, the $E_{\mathrm{p}}$ of each drug and cutting agent is essential to detect the illicit drug and display the result through a userfriendly interface. 


\subsection{Testing the portable electrochemical device with confiscated samples}

It has been shown that the electrochemical profile of cutting agents differs from the profile of most common illicit drugs which is essential to avoid false positives that can induce an incorrect confiscation of a cargo. Nevertheless, it is important for law enforcement to know which illicit compound is present in the suspicious sample. For this reason, the use of suitable conditions (i.e. buffer and SPE) is essential for the detection of the corresponding illicit drug. For example, the oxidation peaks of methamphetamine and MDMA could overlap at $\mathrm{pH} 12$, but $\mathrm{pH} 5$ can confirm the presence of MDMA. Another example is the use of p-SPE for the detection of heroin. To choose the optimal sensing strategy, a preliminary flowchart based on visual identification is included. The flowchart allows for the selection of the best strategy according to the physical appearance of the suspected sample (Figure 1). Hence, when a white powder is encountered, a regular $\mathrm{pH}$ 12 with a SPE would be performed as cocaine is most likely to be encountered. If there is no electrochemical profile, then the strategy with pH 10 and NQS would be performed. In case of finding a brown powder, the $\mathrm{pH} 12$ with p-SPE strategy would be used. Concerning tablets, the sample would be expected to contain MDMA or amphetamine. Therefore, $\mathrm{pH} 5$ with SPE strategy is the best option to detect the single MDMA oxidation peak as the methylenedioxy group of MDMA is electroactive at this $\mathrm{pH}$. If no output from the result is given, pH 10 and NQS would be performed. Finally, if a crystal form is encountered, pH 12 would be used for the detection of methamphetamine and can be complemented by the $\mathrm{pH} 5$ strategy to confirm the presence of MDMA. Overall, the combination of physical appearance and the electrochemical test could be a fast methodology for the classification of illicit drugs in seizures.



Figure 4. On-site detection of illicit drugs with a portable electrochemical device. A) Elements of the electrochemical device (1-potentiostat, 2-buffer tube, 3-SPE, 4-disposable spatula, 5-disposable pipette, 6-confiscated sample); B) Sampling procedure by filling the tip of the disposable spatula from the sample (e.g. powder or oil), adding it to the $15 \mathrm{~mL}$ buffer container, and thoroughly shaking it for $30 \mathrm{~s}$; C) deposition of a drop of the solution on the SPE ready for the electrochemical interrogation; and D) user-friendly interface showing the results of the analysis.

Once the library of electrochemical profiles was incorporated into the database by writing the potential windows from the oxidation of the illicit drugs, the electrochemical test was evaluated with confiscated samples provided by NICC. The electrochemical device consists of a miniaturized potentiostat with Bluetooth ${ }^{\circledR}$ connectivity, a disposable SPE, a sampling container, a disposable spatula, and a disposable pipette (Figure $4 \mathrm{~A}$ ). The sampling procedure consists of collecting the sample (either powder, liquid, or crystal) with the disposable spatula (full tip of the spatula) into a tube containing $15 \mathrm{~mL}$ of the suitable buffer (Figure 4B), previously selected by using the flowchart. After shaking thoroughly, a drop of the solution is deposited on the SPE with the disposable pipette (Figure 
4C). For the analysis of tablets, they are ground into powder form. Following the deposition of the sample solution, the operation is started via the user-friendly interface launching the electrochemical method. After $45 \mathrm{~s}$ in which the SWV is applied, data treatment is executed and the results are subsequently displayed (Figure 4D). For the analysis of confiscated samples, the strategies employing $\mathrm{pH} 12$, p-SPE in $\mathrm{pH} 12, \mathrm{pH}$, and $\mathrm{pH} 10$ with NQS were employed for cocaine and methamphetamine, heroin, MDMA, and amphetamine, respectively.

A) Cocaine samples

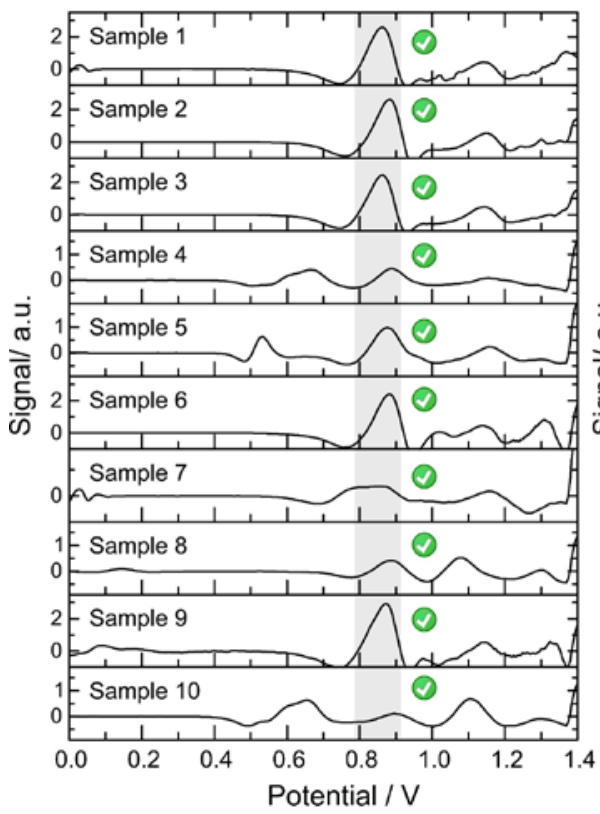

C) MDMA samples

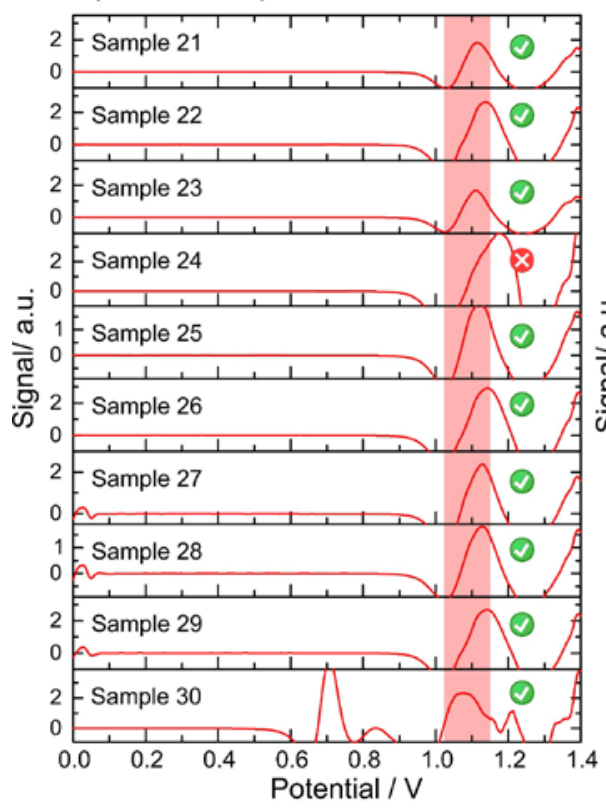

B) Heroin samples

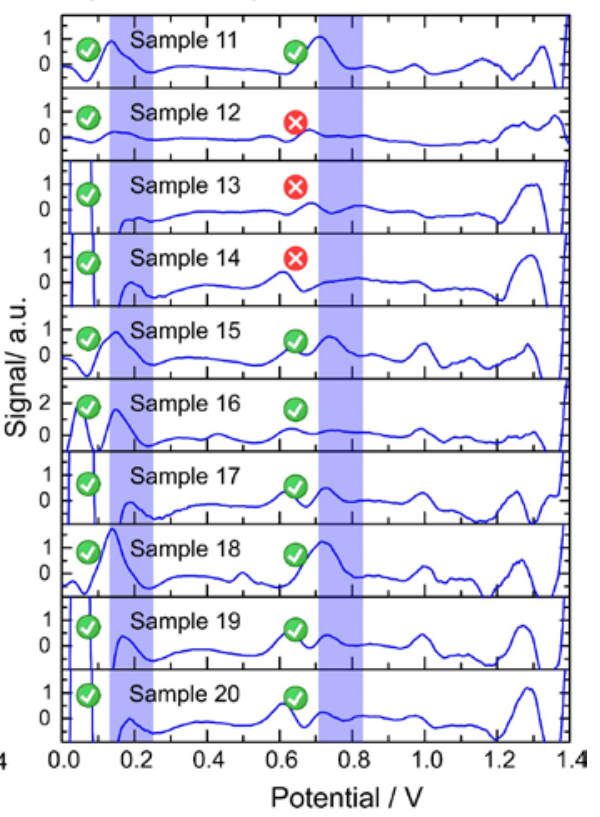

D) Amphetamine samples

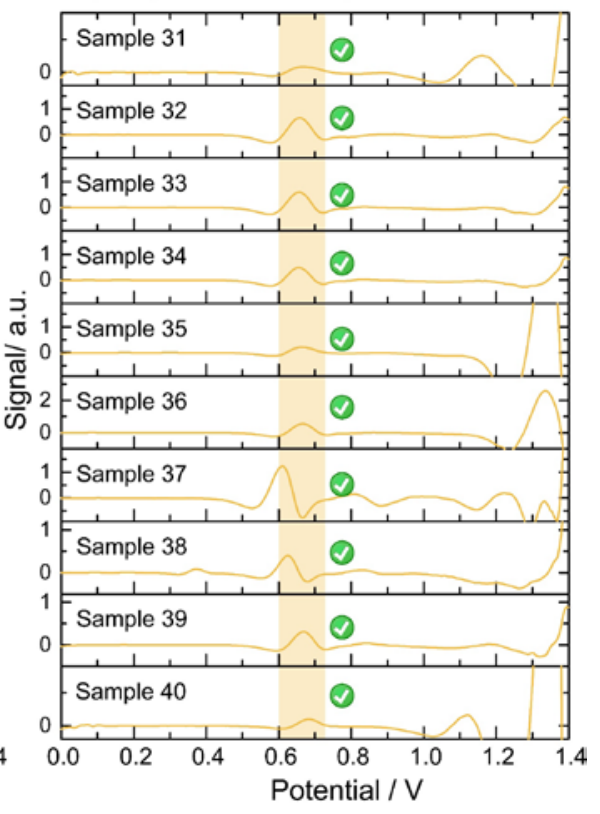

Figure 5. Data output from the script after analysis of the confiscated samples. Electrochemical analysis performed on: A) cocaine samples in phosphate buffer $\mathrm{pH} 12$ using a screen-printed electrode; B) heroin samples in phosphate buffer $\mathrm{pH} 12$ using an anodic pretreated screen-printed electrode; C) MDMA samples in acetate buffer pH 5 using a screen-printed electrode; and D) amphetamine samples in hydrogen carbonate buffer $\mathrm{pH}$ 10 with NQS using a screen-printed electrode. A baseline correction and top-hat filter are applied to the raw data (square-wave voltammograms). 
E) Methamphetamine samples

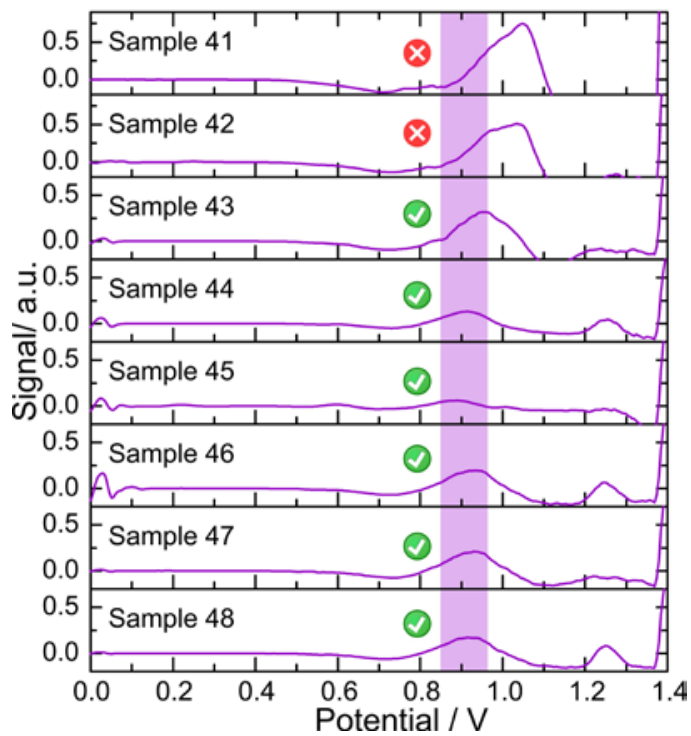

Figure 5 cont. Data output from the script after the analysis of confiscated samples: E) Electrochemical analysis of methamphetamine samples in phosphate buffer $\mathrm{pH} 12$ using a screen-printed electrode. Baseline correction and top-hat filter are applied to the raw data (square-wave voltammograms).

The reliability of the electrochemical device was evaluated in 48 confiscated samples analyzed with the standard method (i.e. GC-MS) by NICC (Table 2). After the electrochemical analysis, 45 out of the 48 samples were positive for the corresponding illicit drug using the described sampling method in comparison to the standard methods. Figure S2 shows the raw data from the SWVs obtained from the electrochemical analysis of the confiscated samples. Figure 5 displays all data output after the application of the script (i.e. baseline correction and top-hat filter). The plots display a sharper peak in comparison to the raw voltammogram which allows narrowing the potential window corresponding to each identification.

The electrochemical test was able to successfully detect all cocaine samples at different concentrations and compositions (Figure 5A). The presence of heroin was also determined in all the samples by the detection of the 6-MAM peak (a degradation product of heroin), as the oxidation peak corresponding to the tertiary amine of heroin was not detected in three tests (Figure 5B). Concerning MDMA, only one sample exhibited an oxidation peak out of the potential window identified for MDMA probably due to the high purity of this specific sample (sample 24 - Figure 5C). In amphetamine samples, the derivatization step with the NQS successfully unraveled the presence of amphetamine which was successfully detected by the script (Figure 5D). Finally, two out of eight methamphetamine samples gave false negatives because the oxidation peak shifted towards higher potentials than the methamphetamine potential window (Figure 5E). This can be avoided by expanding the potential window for the detection of each illicit drug while avoiding overlaps with the potential window of the cutting agents. Figure S3 illustrates the collection of screenshots obtained from the user interface, displaying the output of the electrochemical results for user-friendly interpretation.

To compare with the electrochemical testing strategy, the same samples were also analyzed with a portable Raman spectrometer and a compact ATR-FTIR spectrometer, since these are commonly used methods in border settings. Table 2 shows the comparison between portable techniques and the standard method. The electrochemical reader, portable Raman spectrometer, and compact ATR-FTIR spectrometer exhibited an accuracy of 
$93.8 \%, 58.3 \%$, and $85.4 \%$, respectively. It is worth mentioning that in the methamphetamine analysis with portable Raman spectroscopy, ephedrine was considered as a true positive. In contrast, during the analysis of amphetamine with the Raman device, other similar structures were not considered as true positive. However, if we account for the detection of a primary amine for the portable Raman device in the amphetamine detection, the accuracy of the device increases up to $75 \%$. Two samples analyzed by the Raman device displayed an unknown result in the on-site test but tested positive during the post-analysis with the Opus software. Overall, the electrochemical device outperformed the Raman device, particularly for heroin and amphetamine detection. It is suggested that the lower performance of the Raman device could be attributed to the colored nature of the samples which can produce fluorescence upon laser irradiation and hinder the acquisition of a proper spectrum. Specifically, heroin samples, which are usually encountered as a brownish powder, were not able to be properly classified by the Raman device. Moreover, amphetamine samples were identified with similar structures, which then is difficult to classify in the field by a non-expert in chemistry. Concerning the compact ATR-FTIR spectrometer, the results of the analysis considerably improved in comparison to the Raman device. Moreover, if we consider 6-MAM (a degradation product from heroin) as a true positive for heroin and amphetamine as a true positive for methamphetamine (due to similar structure), the accuracy of the ATR-FTIR improves to $89.6 \%$. This test with confiscated samples demonstrated the flaws of current portable methods and opens the possibility for new electrochemical tests to be implemented into common practices. Therefore, the electrochemical test is positioned as a reliable alternative for its use in the field due to its accuracy, affordability, reliability, and user-friendliness.

Table 2. Results of the analysis by the analytical methods and composition of the confiscated samples.

\begin{tabular}{|c|c|c|c|c|}
\hline $\begin{array}{l}\text { Sam- } \\
\text { ple }\end{array}$ & GC-MS & $\begin{array}{l}\text { Electrochemical } \\
\text { reader }\end{array}$ & Portable Raman & Compact ATR-FTIR \\
\hline 1 & Cocaine & Cocaine & Cocaine mixture & Cocaine $\mathrm{HCl}$ \\
\hline 2 & Cocaine, caffeine, levamisole & Cocaine & Cocaine mixture & Cocaine $\mathrm{HCl}$ \\
\hline 3 & Cocaine, levamisole & Cocaine & Cocaine mixture & Cocaine $\mathrm{HCl}$ \\
\hline 4 & Cocaine, lidocaine, levamisole, phenacetin & Cocaine, lidocaine & Lidocaine, cocaine mixture & Cocaine $\mathrm{HCl}$, lidocaine $\mathrm{HCl}$ \\
\hline 5 & Cocaine, phenacetin, levamisole & $\begin{array}{l}\text { Cocaine, phenace- } \\
\text { tin }\end{array}$ & Cocaine mixture & Cocaine $\mathrm{HCl}$, phenacetin \\
\hline 6 & Cocaine, levamisole & Cocaine & Cocaine mixture & Cocaine $\mathrm{HCl}$ \\
\hline 7 & Cocaine base & Cocaine & $\begin{array}{l}\text { Benzyl benzoate, cocaine mix- } \\
\text { ture }\end{array}$ & Benzoylecgonine \\
\hline 8 & Cocaine, levamisole & $\begin{array}{l}\text { Cocaine, levami- } \\
\text { sole }\end{array}$ & Cocaine mixture & Cocaine, levamisole \\
\hline 9 & Cocaine, caffeine & Cocaine & Cocaine mixture & Cocaine $\mathrm{HCl}$ \\
\hline 10 & Cocaine, lidocaine, levamisole & Cocaine, lidocaine & Lidocaine & Lidocaine base \\
\hline 11 & Heroin, caffeine, 6-MAM, noscapine, papaverine & Heroin & Normorphine, heroin base & Heroin $\mathrm{HCl}$, caffeine \\
\hline 12 & $\begin{array}{l}\text { Heroin, caffeine, 6-MAM, morphine, noscapine, } \\
\text { papaverine }\end{array}$ & Heroin & CTMACl, tin(II) $\mathrm{Cl}$ dihydrate & 6-MAM, caffeine \\
\hline 13 & $\begin{array}{l}\text { Heroin, caffeine, paracetamol, 6-MAM, noscapine, } \\
\text { papaverine }\end{array}$ & $\begin{array}{l}\text { Heroin, paraceta- } \\
\text { mol }^{*}\end{array}$ & CTMACl, tin(II) $\mathrm{Cl}$ dihydrate & Heroin, paracetamol, caffeine \\
\hline 14 & $\begin{array}{l}\text { Heroin, caffeine, 6-MAM, noscapine, papaverine, } \\
\text { paracetamol }\end{array}$ & Heroin & Unknown (lead tin, CTMACl) & Paracetamol, caffeine \\
\hline 15 & Heroin, 6-MAM, noscapine, papaverine & Heroin & CTMACl & Heroin, noscapine \\
\hline 16 & $\begin{array}{l}\text { Heroin, paracetamol, 6-MAM, morphine, caffeine, codeine, } \\
\text { noscapine, papaverine }\end{array}$ & $\begin{array}{l}\text { Heroin, paraceta- } \\
\text { mol }\end{array}$ & $\begin{array}{l}\text { CTMACl, a-pyrrolidinohex- } \\
\text { anophenone }\end{array}$ & Heroin, noscapine \\
\hline 17 & $\begin{array}{l}\text { Heroin, paracetamol, 6-MAM, caffeine, noscapine, } \\
\text { papaverine }\end{array}$ & $\begin{array}{l}\text { Heroin, paraceta- } \\
\text { mol }\end{array}$ & CTMACl, tin(II) $\mathrm{Cl}$ dihydrate & Heroin, paracetamol, caffeine \\
\hline 18 & $\begin{array}{l}\text { Heroin, paracetamol, 6-MAM, caffeine, noscapine, } \\
\text { papaverine }\end{array}$ & Heroin & Unknown (heroin base) & Heroin $\mathrm{HCl}$, caffeine \\
\hline 19 & $\begin{array}{l}\text { Heroin, paracetamol, 6-MAM, caffeine, noscapine, } \\
\text { papaverine }\end{array}$ & $\begin{array}{l}\text { Heroin, paraceta- } \\
\text { mol }\end{array}$ & CTMACl & $\begin{array}{l}\text { Heroin, caffeine, paracetamol, } \\
\text { noscapine }\end{array}$ \\
\hline 20 & $\begin{array}{l}\text { Heroin, paracetamol, 6-MAM, caffeine, noscapine, } \\
\text { papaverine }\end{array}$ & $\begin{array}{l}\text { Heroin, paraceta- } \\
\text { mol }\end{array}$ & CTMACl, tin(II) $\mathrm{Cl}$ dihydrate & Heroin, paracetamol, caffeine \\
\hline 21 & MDMA & MDMA & MDMA tablet & MDMA \\
\hline
\end{tabular}


MDMA

MDMA

MDMA

MDMA

MDMA

MDMA

MDMA

MDMA

MDMA

Amphetamine

Amphetamine

Amphetamine

Amphetamine

Amphetamine, caffeine

Amphetamine, caffeine

Amphetamine

Amphetamine

Amphetamine

Amphetamine, caffeine

Methamphetamine

Methamphetamine

Methamphetamine

Methamphetamine base

Methamphetamine

Methamphetamine

Methamphetamine

Methamphetamine

MDMA
MDMA
Negative
MDMA
MDMA
MDMA
MDMA
MDMA
MDMA

Amphetamine

Amphetamine

Amphetamine

Amphetamine

Amphetamine

Amphetamine

Amphetamine

Amphetamine

Amphetamine

Negative

Negative

Methamphetamine

Methamphetamine

Methamphetamine

Methamphetamine

Methamphetamine

Methamphetamine
Amphetamine

\begin{tabular}{|c|c|}
\hline MDMA crystals & MDMA \\
\hline MDMA tablet & MDMA \\
\hline MDMA crystals & MDMA \\
\hline MDMA tablet & MDMA \\
\hline MDMA tablet & MDMA \\
\hline MDMA crystals & MDMA \\
\hline MDMA crystals & MDMA \\
\hline MDMA crystals & MDMA \\
\hline MDMA crystals & Euthylon, safrole \\
\hline Paraform, $\mathrm{KH}_{2} \mathrm{PO}_{4}$ & Lactose, amphetamine \\
\hline Norephedrine $\mathrm{HCl}$ & Amphetamine, saccharose \\
\hline $\begin{array}{c}\text { Desoxy-D2PM, norephedrine } \\
\mathrm{HCl}\end{array}$ & Amphetamine \\
\hline $\begin{array}{c}\text { Desoxy-D2PM, norephedrine } \\
\mathrm{HCl}\end{array}$ & Amphetamine \\
\hline Amphetamine & Caffeine, amphetamine \\
\hline $\begin{array}{c}\text { Unknown (deltamethrin, 2- } \\
\text { phenylethylamine) }\end{array}$ & di-amphetamine, caffeine \\
\hline $\begin{array}{l}\text { 1-phenyl-1-propanol, 2-phe- } \\
\text { nylethylamine }\end{array}$ & Amphetamine oil \\
\hline $\begin{array}{l}\text { 1-phenyl-1-propanol, 2-phe- } \\
\text { nylethylamine }\end{array}$ & Amphetamine oil \\
\hline $\begin{array}{l}\text { 1-phenyl-1-propanol, B- } \\
\text { methylphenethylamine }\end{array}$ & Amphetamine \\
\hline Amphetamine & Caffeine \\
\hline $\begin{array}{l}\text { 1,2-diphenyl-2-propanol, trytil } \\
\text { alcohol }\end{array}$ & Methamphetamine \\
\hline Ephedrine $\mathrm{HCl}$ & Methamphetamine \\
\hline Ephedrine $\mathrm{HCl}$ & Methamphetamine \\
\hline $\begin{array}{l}\text { 1-phenyl-1-propanol, 2-phe- } \\
\text { nylethylamine }\end{array}$ & Methamphetamine \\
\hline $\begin{array}{l}\text { 1-phenyl-1-propanol, pen- } \\
\text { tylbenzene }\end{array}$ & BMK, amphetamine oil \\
\hline Ephedrine $\mathrm{HCl}$ & Methamphetamine \\
\hline Ephedrine $\mathrm{HCl}$ & Methamphetamine \\
\hline Ephedrine $\mathrm{HCl}$ & Methamphetamine \\
\hline
\end{tabular}

MDMA

MDMA

MDMA

MDA

MDMA

Euthylon, safrole

Lactose, amphetamine

Amphetamine

Amphetamine

phetamine

Amphetamine oil

mphetamine

Methamphetamine

hamphetamine

Methamphetamine

Abbreviations: 6-MAM=6-Monoacetylmorphine; ATR-FTIR= attenuated total reflectance-Fourier transform infrared; $\mathrm{BMK}=$ benzyl methyl ketone; $\mathrm{CTMACl}=$ Cetyltrimethylammonium chloride; Desoxy-D2PM=1-(S)-2-diphenylmethylpyrrolidine $\mathrm{HCl}$; $\mathrm{KH}_{2} \mathrm{PO}_{4}=$ potassium dihydrogen phosphate; MDMA=3,4-methylenedioxymethamphetamine. *Double test with more loading of the sample. Red indicates a false negative result. Orange color indicates a false positive result from a structurarly similar compounds than the illicit drug.

\section{Conclusions}

In this work, the analysis of confiscated samples from illicit drugs is presented by the use of a portable electrochemical device. First, the construction of a library from several electrochemical profiles of standards of illicit drugs and common cutting agents at different conditions at SPEs is performed. After the selection of the suitable conditions and the integration of the potential windows according to $E_{\mathrm{p}}$ values for each target into a tailormade script, the electrochemical device is ready for the on-site analysis. The examination of 48 confiscated samples with the electrochemical device, a portable Raman spectrometer, and a compact ATR-FTIR spectrometer showed an outstanding performance of the electrochemical device in front of the Raman device and the compact ATR-FTIR (accuracy of $93.8 \%, 58.3 \%$, and $85.4 \%$ respectively) according to the GC-MS identification provided by a forensic institute. Overall, the electrochemical device based on SPEs is presented as 
a promising alternative to current rapid and on-site methods for the detection of illicit drugs at border and coast controls.

Author Contributions: Conceptualization, M.P.; methodology, M.P., A.S. N.FM.; software, R.VE.; validation, M.P., A.S., and F.VD; M.P.; investigation, M.P.; resources, K.DW. and F.D.; data curation, M.P. and A.S.; writing - original draft preparation, M.P.; writing - review and editing, M.P., A.S., R.VE, N.FM, A.L.; visualization, M.P.; supervision, K.DW.; project administration, K.DW.; funding acquisition, K.DW. All authors have read and agreed to the published version of the manuscript.

Funding: This research was funded by European Union's Horizon 2020 research and innovation programme, grant number 833787, Bordersens.

Conflicts of Interest: The authors declare no conflict of interest. The funders had no role in the design of the study; in the collection, analyses, or interpretation of data; in the writing of the manuscript, or in the decision to publish the results.

\section{References}

1. European Monitoring Centre for Drugs and Drug Addiction (EMCDDA) European Drug Report 2021: Trends and Developments; Publications Office of the European Union, 2021;

2. $\quad$ United Nations Office on Drugs and Crime World Drug Report 2021; 2021;

3. European Monitoring Centre for Drugs and Drug Addiction and Europol EU Drug Markets: Impact of COVID-19; 2020; ISBN 9789294974938.

4. Philp, M.; Fu, S. A review of chemical 'spot' tests: A presumptive illicit drug identification technique. Drug Test. Anal. 2018, 10, 95-108, doi:10.1002/dta.2300.

5. Harper, L.; Powell, J.; Pijl, E.M. An overview of forensic drug testing methods and their suitability for harm reduction pointof-care services. Harm Reduct. J. 2017, 14, 52, doi:10.1186/s12954-017-0179-5.

6. Katainen, E.; Elomaa, M.; Laakkonen, U.M.; Sippola, E.; Niemelä, P.; Suhonen, J.; Järvinen, K. Quantification of the amphetamine content in seized street samples by Raman spectroscopy. J. Forensic Sci. 2007, 52, 88-92, doi:10.1111/j.15564029.2006.00306.x.

7. De Oliveira Penido, C.A.F.; Pacheco, M.T.T.; Lednev, I.K.; Silveira, L. Raman spectroscopy in forensic analysis: Identification of cocaine and other illegal drugs of abuse. J. Raman Spectrosc. 2016, 47, 28-38, doi:10.1002/jrs.4864.

8. Kranenburg, R.F.; Verduin, J.; Ridder, R.; Weesepoel, Y.; Alewijn, M.; Heerschop, M.; Keizers, P.H.J.; Esch, A.; Asten, A.C. Performance Evaluation of Handheld Raman Spectroscopy for Cocaine Detection in Forensic Case Samples. Drug Test. Anal. 2020, 13, 1054-1067, doi:10.1002/dta.2993.

9. Kranenburg, R.F.; Verduin, J.; Weesepoel, Y.; Alewijn, M.; Heerschop, M.; Koomen, G.; Keizers, P.; Bakker, F.; Wallace, F.; van Esch, A.; et al. Rapid and robust on-scene detection of cocaine in street samples using a handheld near-infrared spectrometer and machine learning algorithms. Drug Test. Anal. 2020, 12, 1404-1418, doi:10.1002/dta.2895.

10. Deconinck, E.; Aït-Kaci, C.; Raes, A.; Canfyn, M.; Bothy, J.L.; Duchateau, C.; Mees, C.; De Braekeleer, K.; Gremaux, L.; Blanckaert, P. An infrared spectroscopic approach to characterise white powders, easily applicable in the context of drug checking, drug prevention and on-site analysis. Drug Test. Anal. 2021, 13, 679-693, doi:10.1002/dta.2973.

11. Dragan, A.M.; Parrilla, M.; Feier, B.; Oprean, R.; Cristea, C.; De Wael, K. Analytical techniques for the determination of Amphetamine-type substances in different matrices: a comprehensive review. Trends Anal. Chem. 2021, 145, 116447, doi:10.1016/j.trac.2021.116447.

12. De Jong, M.; Florea, A.; Eliaerts, J.; Van Durme, F.; Samyn, N.; De Wael, K. Tackling Poor Specificity of Cocaine Color Tests by Electrochemical Strategies. Anal. Chem. 2018, 90, 6811-6819, doi:10.1021/acs.analchem.8b00876.

13. Shanmugam, S.T.; Echelpoel, R. Van; Boeye, G. Towards Developing a Screening Strategy for Ecstasy: Revealing the Electrochemical Profile. ChemElectroChem 2021, 8, 4826-4834, doi:10.1002/celc.202101198. 
14. Gerace, E.; Seganti, F.; Luciano, C.; Lombardo, T.; Di Corcia, D.; Teifel, H.; Vincenti, M.; Salomone, A. On-site identification of psychoactive drugs by portable Raman spectroscopy during drug-checking service in electronic music events. Drug Alcohol Rev. 2019, 38, 50-56, doi:10.1111/dar.12887.

15. de Araujo, W.R.; Cardoso, T.M.G.; da Rocha, R.G.; Santana, M.H.P.; Muñoz, R.A.A.; Richter, E.M.; Paixão, T.R.L.C.; Coltro, W.K.T. Portable analytical platforms for forensic chemistry: A review. Anal. Chim. Acta 2018, 1034, 1-21, doi:10.1016/j.aca.2018.06.014.

16. Zanfrognini, B.; Pigani, L.; Zanardi, C. Recent advances in the direct electrochemical detection of drugs of abuse. J. Solid State Electrochem. 2020, 24, 2603-2616, doi:10.1007/s10008-020-04686-z.

17. De Rycke, E.; Stove, C.; Dubruel, P.; De Saeger, S.; Beloglazova, N. Recent developments in electrochemical detection of illicit drugs in diverse matrices. Biosens. Bioelectron. 2020, 169, 112579, doi:10.1016/j.bios.2020.112579.

18. Shaw, L.; Dennany, L. Applications of electrochemical sensors: Forensic drug analysis. Curr. Opin. Electrochem. 2017, 3, 2328, doi:https://doi.org/10.1016/j.coelec.2017.05.001.

19. Teymourian, H.; Parrilla, M.; Sempionatto, J.R.; Montiel, N.F.; Barfidokht, A.; Van Echelpoel, R.; De Wael, K.; Wang, J. Wearable Electrochemical Sensors for the Monitoring and Screening of Drugs. ACS Sensors 2020, 5, 2679-2700, doi:10.1021/acssensors.0c01318.

20. Parrilla, M.; Joosten, F.; De Wael, K. Enhanced electrochemical detection of illicit drugs in oral fluid by the use of surfactantmediated solution. Sensors Actuators B Chem. 2021, 348, 130659, doi:10.1016/j.snb.2021.130659.

21. Parrilla, M.; Slosse, A.; Van Echelpoel, R.; Felipe Montiel, N.; Van Durme, F.; De Wael, K. Portable Electrochemical Detection of Illicit Drugs in Smuggled Samples: Towards More Secure Borders. Chem. Proc. 2021, 5, 44, doi:10.3390/CSAC2021-10612.

Freitas, J.M.; Ramos, D.L.O.; Sousa, R.M.F.; Paixão, T.R.L.C.; Santana, M.H.P.; Muñoz, R.A.A.; Richter, E.M. A portable electrochemical method for cocaine quantification and rapid screening of common adulterants in seized samples. Sensors Actuators, B Chem. 2017, 243, 557-565, doi:10.1016/j.snb.2016.12.024.

23. Schram, J.; Parrilla, M.; Sleegers, N.; Samyn, N.; Bijvoets, S.M.; Heerschop, M.W.J.; van Nuijs, A.L.N.; De Wael, K. Identifying Electrochemical Fingerprints of Ketamine with Voltammetry and Liquid Chromatography-Mass Spectrometry for Its Detection in Seized Samples. Anal. Chem. 2020, 92, 13485-13492, doi:10.1021/acs.analchem.0c02810.

24. Felipe Montiel, N.; Parrilla, M.; Beltrán, V.; Nuyts, G.; Van Durme, F.; De Wael, K. The opportunity of 6-monoacetylmorphine to selectively detect heroin at preanodized screen printed electrodes. Talanta 2021, 226, 122005, doi:10.1016/j.talanta.2020.122005.

25. Schram, J.; Parrilla, M.; Sleegers, N.; Van Durme, F.; van den Berg, J.; van Nuijs, A.L.N.; De Wael, K. Electrochemical profiling and LC-MS characterization of synthetic cathinones : from methodology to detection in forensic samples. Drug Test. Anal. 2021, 13, 1282-1294, doi:10.1002/dta.3018.

26. Smith, J.P.; Metters, J.P.; Khreit, O.I.G.; Sutcliffe, O.B.; Banks, C.E. Forensic electrochemistry applied to the sensing of new psychoactive substances: Electroanalytical sensing of synthetic cathinones and analytical validation in the quantification of seized street samples. Anal. Chem. 2014, 86, 9985-9992, doi:10.1021/ac502991g.

27. Parrilla, M.; Montiel, N.F.; Van Durme, F.; De Wael, K. Derivatization of amphetamine to allow its electrochemical detection in illicit drug seizures. Sensors Actuators B. Chem. 2021, 337, 129819, doi:10.1016/j.snb.2021.129819.

28. Slosse, A.; Van Durme, F.; Samyn, N.; Mangelings, D.; Vander Heyden, Y. Evaluation of data preprocessings for the comparison of GC-MS chemical profiles of seized cannabis samples. Forensic Sci. Int. 2020, 310, 110228, doi:10.1016/j.forsciint.2020.110228.

29. Van Echelpoel, R.; de Jong, M.; Daems, D.; Van Espen, P.; De Wael, K. Unlocking the full potential of voltammetric data analysis: A novel peak recognition approach for (bio)analytical applications. Talanta 2021, 233, 122605, doi:10.1016/j.talanta.2021.122605. 
30. Moro, G.; Barich, H.; Driesen, K.; Felipe Montiel, N.; Neven, L.; Mendonça, C.D.; Shanmugam, S.T.; Daems, E.; Wael, K. De Unlocking the full power of electrochemical fingerprinting for on-site sensing applications. Anal. Bioanal. Chem. 2020, 412, 5955-5968, doi:10.1007/s00216-020-02584-x.

31. Milhazes, N.; Martins, P.; Uriarte, E.; Garrido, J.; Calheiros, R.; Marques, M.P.M.; Borges, F. Electrochemical and spectroscopic characterisation of amphetamine-like drugs: Application to the screening of 3,4-methylenedioxymethamphetamine (MDMA) and its synthetic precursors. Anal. Chim. Acta 2007, 596, 231-241.

32. Broséus, J.; Gentile, N.; Esseiva, P. The cutting of cocaine and heroin: A critical review. Forensic Sci. Int. 2016, 262, 73-83, doi:10.1016/j.forsciint.2016.02.033.

33. Fiorentin, T.R.; Krotulski, A.J.; Martin, D.M.; Browne, T.; Triplett, J.; Conti, T.; Logan, B.K. Detection of Cutting Agents in Drug-Positive Seized Exhibits within the United States. J. Forensic Sci. 2019, 64, 888-896, doi:10.1111/1556-4029.13968. 